\title{
Détermination des hauteurs d'eau extrêmes le long des côtes de France
}

par

\author{
M. Bernard SIMON \\ Ingénieur Civil
}

Établissement Principal du Service Hydrographique et Océanographique de la Marine

\section{Résumé}

Statistique des niveaux marins extrêmes le long des côtes de France :

Le niveau de la mer résulte de la superposition de deux phénomènes largement indépendants : la marée astronomique et les surcotes-décotes d'origine essentiellement métérologique. Si l'on dispose d'observations suffisantes, il peut être aisé de déterminer les probabilités d'occurence d'événements rares tels que les vives-eaux exceptionnelles ou les surcotes importantes, mais leur coüncidence peut être si rare qu'elle n'a jamais été observée. Pourtant, sa probabilité peut être calculée à partir de la connaissance des lois de probabilité liées à la marée d'une part et aux surcotes d'autre part.

Ce travail a été effectué pour quinze ports de France où plus de dix années d'observations de marée sont disponibles. Les résultats se présentent sous forme de relations entre hauteurs extrêmes et périodes de retour.

Pour les sites beaucoup plus nombreux où les observations sont de courte durée, les lois de répartitions des surcotes ont été établies à partir des relations existant entre les surcotes de ports voisins.

Enfin la densité d'observatoires étant généralement suffisante, il a été possible, par interpolations, d'étendre les résultats à tous les points des côtes de France de I'Atlantique et de La Manche. Ils se présentent sous forme de cartes montrant les lignes d'égales hauteurs atteintes par la mer avec une période de retour donnée. Les incertitudes associées montrent des zones où des observations complémentaires sont requises.

\section{Introduction}

Quel est le niveau maximum atteint par la mer ? Cette question se pose pour des raisons évidentes de sécurité et d'optimisation des coûts à l'occasion de constructions d'ouvrages portuaires, de digues de protection ou d'équipements industriels tels que les centrales électronucléaires ou les plates-formes de forage en mer. Mais c'est pour 
des raisons juridiques, dans le cadre de l'étude d'une proposition de loi relative à la délimitation du Domaine Public Maritime, que le SHOM a eté sollicité afin de donner une réponse. En effet, selon une ordonnance de COLBERT, le domaine maritime est défini comme la limite atteinte par la mer lors du "grand flot de mars" ; cette définition n'est pas rigoureuse car deux opérations de délimitation sont susceptibles de donner des résultats différents.

\section{Contexte bibliographique}

Très souvent les estimations de niveaux extrêmes sont faites à partir des maximums annuels (Lennon, 1963 ; Graff, 1981). Il est relativement aisé de déterminer une période de retour dont la valeur est faible, comparée à la durée du phénomène observé. Un simple calcul de moyenne suffit et l'intervalle de confiance est d'autant plus etroit que le nombre d'observations est important. Mais cette methode ne convient pas lorsque les durées d'observations ne sont pas d'un ordre de grandeur supérieur aux périodes de retour recherchées.

En géneral, on cherche à ajuster les fonctions de distributions à des lois destinées à permettre l'extrapolation pour des périodes de retour importantes (Walden \& Prescott, 1980 ; Middleton et Thomson, 1986). Mais ces méthodes se heurtent à certaines difficultés. Pour les besoins de l'extrapolation vers des valeurs de période de retour supérieures à la duree d'observation, ce sont évidemment les valeurs observées les plus elevées qui présentent le plus d'intérêt pour déterminer les paramètres de cette loi mais ce sont précisément les observations correspondantes qui sont les moins nombreuses et présentent une dispersion importante les rendant souvent inexploitables.

Une autre difficulté à laquelle se sont heurtés tous les auteurs est la nécessité de valider avec soin ces valeurs extrêmes, parmi lesquelles la proportion de valeur erronées est parfois très importante.

Les premières études sur l'estimation des niveaux extrêmes de la mer se sont inspirées des travaux sur l'estimation des probabilités de crue des rivières (Lennon, 1963 ; Suthons, 1963).

En 1978, Pugh et Vassie tirent profit du fait que le niveau de la mer est la superposition de deux phénomènes largement indépendants, la marée astronomique et les effets météorologiques. Cette approche présente un grand intérêt car elle permet d'accéder à l'estimation des périodes de retour d'événements très rares sans que le choix du modèle d'extrapolation ne s'avère critique.

Il est par exemple possible d'estimer les probabilités d'apparition d'evénements rares sans être exceptionnels telles que les hauteurs théoriques des grandes vives eaux ou les surcotes importantes. Mais la conjonction de deux de ces événements peut être si peu probable qu’elle correspond à des périodes de retour bien supérieures à la durée des observations analysées.

Cette idé va être exploitee pour l'étude des niveaux exceptionnels sur les côtes de France; mais la méthode de Pugh et Vassie reprise et complétée par Tawn et Vassie 
(1989, 1990, 1991), qui consiste à analyser les hauteurs observées heure par heure n'a pas été retenue car elle présente deux inconvénients importants :

- les surcotes-décotes aux heures consécutives sont très corrélées, ce qui complique leur traitement statistique ;

- les defauts éventuels de calage en heure des observations (ou des prédictions) se traduisent par des surcotes-décotes, importantes au moment des mi-marées, qui risquent de fausser très sensiblement les résultats.

Ces difficultés sont en grande partie évitées si l'on se limite à l'analyse des écarts entre les hauteurs de pleines mers observées et prédites.

\section{Description de la méthode}

La méthode retenue consiste en la combinaison des lois de probabilités des hauteurs de pleines mers prédites et des valeurs des surcotes observées.

\subsection{Marée prédite}

Le caicul de la marée astronomique est une activité traditionnelle du Service Hydrographique et Océanographique de la Marine. On estime habituellement, lorsque la marée est importante comme c'est le cas sur les côtes de France, qu'il est nécessaire d'analyser une anné d'observations pour obtenir une précision des prédictions suffisante pour les besoins de la navigation, une précision quasi optimale pouvant être obtenue si l'on dispose de vingt années d'observations. Cette dernière condition est remplie pour une dizaine de ports des côtes de France. Pour ceux-ci, la question de la précision de la prédiction ne se posera donc pas en pratique. Ces ports seront appelés par la suite, ports de référence.

Lors des sondages hydrographiques, il est nécessaire de connaître la hauteur de la marée à l'endroit et au moment du sondage afin de rapporter les hauteurs mesurées au niveau de référence des cartes marines (zéro hydrographique). Ce besoin spécifique a exigé la mise au point de méthodes de calcul de la marée en tout point du plateau continental (Simon, 1988). Par grands fonds la correction de marée est négligée.

C'est un problème difficile qui fait encore l'objet d'etudes, mais on peut estimer actuellement que la précision du calcul de la marée astronomique en tout point des côtes de France (à l'exciusion des zones d'estuaires) est satisfaisante pour les besoins de l'hyđrographie grâce aux résultats de modèles, à l'exploitation des mesures des marégraphes à capteur de pression immergés au large (Simon, 1991) et à l'utilisation des moyens de calcul modernes.

Par ailleurs, le problème de la précision de la prédiction n'est pas réellement critique puisque les defauts éventuels de celle-ci seront pris en compte dans les surcotesdécotes et analysés comme telles. 


\subsection{Surcotes-décotes, ports de référence}

Les surcotes-décotes sont essentiellement dues aux influences météorologiques. C'est un signal aléatoire qui devra donc être traité à l'aide de méthodes statistiques. A une hauteur d'eau donnee, correspond l'ensemble des couples marée prédite-surcote dont la somme est égale à cette hauteur.

Si les deux phénomènes sont indépendants, la probabilité attachée à l'observation d'une hauteur d'eau supérieure à une valeur donnée se déduit facilement des probabilités attachees d'une part aux hauteurs de pleines-mers prédites, d'autre part à la répartition des surcotes (proportion des surcotes supérieures à une valeur donnée).

La difficulté provient du fait que les surcotes très importantes, donc très rares ne peuvent pas être négligées a priori. On est ainsi amené à prendre en compte des probabilités d'apparition de surcotes qui n'ont jamais été observées et donc à choisir un modèle d'extrapolation.

Après des essais d'ajustement à quelques lois classiques, utilisées notamment dans l'estimation des probabilités de crues, la loi de Gumbel, 1958, a été adoptée. D'autres lois pourraient éventuellement convenir, mais il s'avère que ce choix n'est pas critique pour les ports où plus de 10 annees d'observations sont disponïbles. C'est le cas pour une quinzaine de ports des côtes de France. Pour ceux-ci, il est donc adimis que tous les éléments nécessaires à l'évaluation des probabilités d'observation des hauteurs de pleînes mers sont disponibles.

\subsection{Surcotes-décotes, ports secondaires}

L'évolution de la répartition des surcotes aux points de mesures où peu d'observations sont disponibles pose un problème très différent car il peut être impossible dans ce cas d'ajuster une loi. La méthode retenue s'appuie sur l'idé qu'il existe évidemment une relation entre les surcotes de ports voisins. L'évaluation des corrélations avec les surcotes observées à l'aide des observations simultanees faites dans les ports importants (où la loi de répartition a pu être établie) devrait donc permettre de résoudre ce problème.

Ce travail a éte réalisé pour tous les points où sont disponibles des observations d'une durée minimale de 1 mois en adoptant pour chacun d'entre eux le port de référence le plus proche pour lequel des observations simultanés sont disponibles.

\subsection{Surcotes-décotes, interpolation}

Compte tenu de la variabilité spatiale des paramètres étudiés (surcote et marée), la densité de points de mesure est a priori satisfaisante (à l'exception de la côte des Landes) pour permettre leur évaluation par interpolation en tout point de la côte. Cependant, la validité des résultats devra être évaluée en tenant compte d'une part, du niveau de corrélation entre les surcotes de ports voisins et d'autre part, de l'incertitude liée aux interpolations. 


\section{Résultats}

\subsection{Observations dans les ports de référence}

Le tableau 1 présente les mesures disponibles aux ports de référence.

Tableau 1 : observations dans les ports de reférence

\begin{tabular}{|l|c|c|c|c|}
\hline \multicolumn{1}{|c|}{ Port } & Début & Fin & $\begin{array}{c}\text { Durée utile } \\
\text { (ans) }\end{array}$ & $\begin{array}{c}\text { Lacunes } \\
(\%)\end{array}$ \\
\hline Dunkerque & 07.06 .1956 & 03.06 .1992 & 28,1 & 21,9 \\
\hline Calais & 16.02 .1965 & 24.12 .1992 & 20,1 & 28,0 \\
\hline Boulogne & 08.09 .1973 & 27.11 .1991 & 15,3 & 15,8 \\
\hline Dieppe & 01.01 .1954 & 04.08 .1989 & 31,1 & 12,7 \\
\hline Le Havre & 18.09 .1961 & 02.09 .1991 & 18,2 & 8,8 \\
\hline Cherbourg & 02.01 .1963 & 06.05 .1992 & 17,0 & 42,0 \\
\hline Saint-Malo & 05.11 .1850 & 30.04 .1917 & 38,7 & 41,8 \\
\hline Roscoff & 07.04 .1973 & 15.12 .1992 & 18,5 & 6,2 \\
\hline Le Conquet & 22.12 .1970 & 04.01 .1993 & 21,0 & 4,7 \\
\hline Brest & 03.05 .1860 & 01.12 .1992 & 158,4 & 8,9 \\
\hline Port-Tudy & 11.08 .1966 & 01.01 .1992 & 17,4 & 31,4 \\
\hline Saint-Nazaire & 16.05 .1957 & 31.12 .1988 & 21,4 & 32,2 \\
\hline Saint-Gildas & 01.08 .1967 & 31.12 .1988 & 20,5 & 4,3 \\
\hline Pointe-de-Grave & 19.04 .1959 & 31.12 .1977 & 13,0 & 30,6 \\
\hline Le Boucau & 23.05 .1967 & 05.01 .1992 & 19,4 & 21,4 \\
\hline
\end{tabular}

\subsection{Exemple du port de Brest}

La figure 1 présente, à titre d'exemple, la densité de probabilité des hauteurs de pleines mers prédites pour Brest. Ce sont les probabilités de prédiction d'une hauteur donnée, à $1 \mathrm{~cm}$ près.

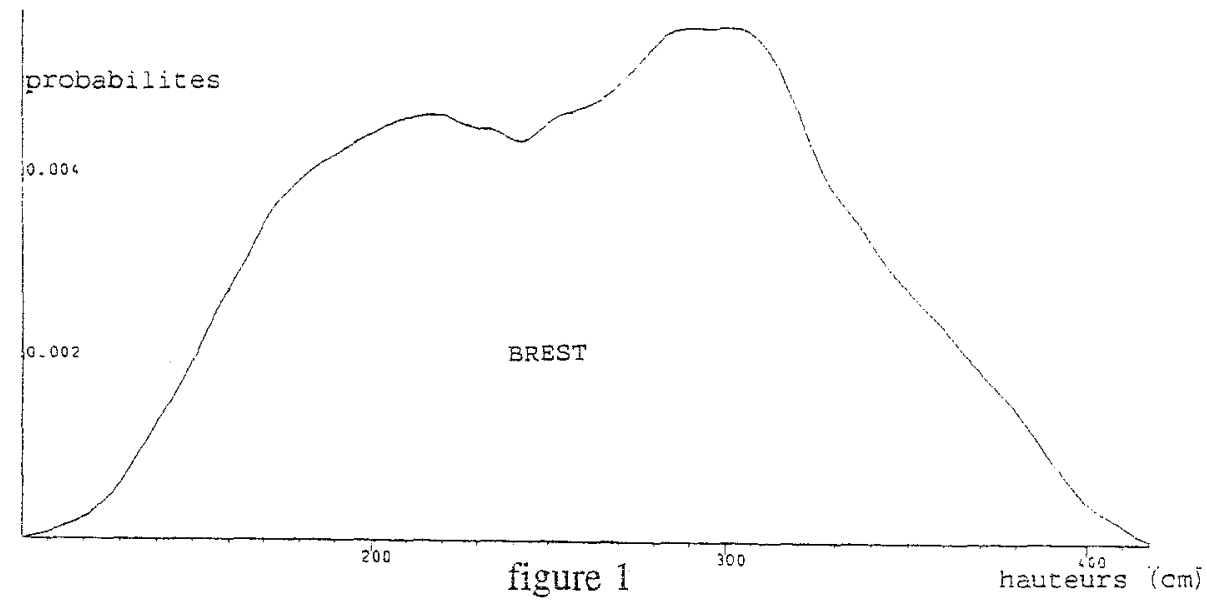




\subsection{Loi de répartition des surcotes}

La figure 2 présente, pour Brest, les taux de surcotes supérieures à une valeur donnée dans un système de coordonnées tel que si la loi de Gumbel était vérifiée, les points expérimentaux seraient parfaitement alignés.

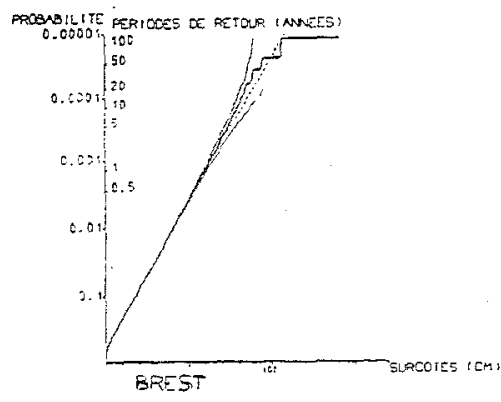

figure 2

En trait discontinu est représentée la droite s'ajustant au mieux aux points expérimentaux selon une régression par moindres carrés.

Les courbes en traits fins représentent les limites entre lesquelles doivent se situer $90 \%$ des points de mesures d'après les hypothèses suivantes:

- la droite de régression représente effectivement la loi de répartition des surcotes;

- le fait, pour une surcote d'être superieure à une valeur donnée est un processus de Poisson.

La loi de Gumbel est assez bien adaptée au problème des surcotes puisque pour tous les ports, les courbes expérimentales sont situées à l'intérieur des limites de confiance à $90 \%$. Cependant, pour les valeurs élevées des surcotes, certaines tendances qui peuvent paraître systématiques sembleraient suggérer qu'une autre loi serait mieux adaptée. Mais il n'a pas semblé utile de rechercher une possible meilleure adaptation, en raison essentiellement du peu de sensibilité de la loi de repartition des hauteurs de pleines mers à la loi de répartition des surcotes pour les valeurs élevées de celles-ci.

\subsection{Ports secoridaires}

Nous supposerons qu'il existe une relation linéaire entre les surcotes en deux ports au moment des pleines mers, cette relation étant représentée par la droite passant au mieux (au sens des moindres carrés) dans le nuage de points. A cette droite de régression, est attaché un degré de confiance lié à la dispersion plus ou moins grande des points de part et d'autre de la droite. Ce degré de confiance est mesuré par un coefficient qque nous appellerons coefficient de détermination variant entre les valeurs 1 lorsque tous les points sont parfaitement alignes et 0 lorsque la droite est complètement indéterminée (points uniformément répartis autour d'un point dont les coordonnees sont les surcotes moyennes en chacun des ports).

Le coefficient de détermination sera utilisé pour repérer les zones où des observations complémentaires sont requises. 


\subsection{Indépendance des surcotes}

Le calcul des périodes de retour des hauteurs de pleines mers suppose que les surcotes soient indépendantes des hauteurs de maré prédites. Le test d'indépendance est effectué en calculant les variances des surcotes pour diverses classes de hauteurs de pleines mers. Aucune dependance n'étant détectée, nous supposerons que l'hypothèse d'indépendance est vérifiée en tout point des côtes de France.

\subsection{Périodes de retour des hauteurs observées}

La figure 3 présente, pour Brest, à titre d'exemple, les périodes de retour correspondant aux hauteurs observées de pleine mer supérieures à la hauteur maximale prédite.

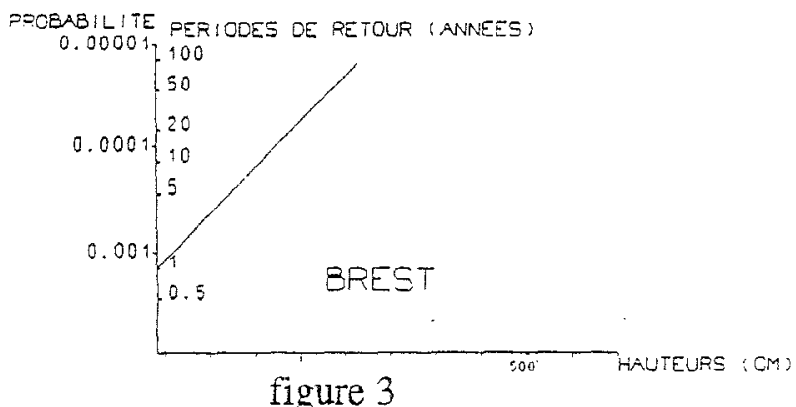

Ces calculs ont été effectués également pour les ports secondaires. Les lois de répartitions des surcotes ont été calculées pour chaque port secondaire à partir des lois de répartition des surcotes aux ports de référence et des relations entre les surcotes aux ports de référence et aux ports secondaires-dont le principe de calcul est exposé au chapitre 4.4 .

\subsection{Lignes d'égales hauteurs de pleines mers}

La méthode exposée ci-dessus a servi à calculer les hauteurs des pleines mers, rapportées au système IGN 69 des altitudes normales correspondant à diverses périodes de retour : $5,10,15,20,25,30,50,75$ et 100 ans.

Les valeurs de hauteurs de pleine mer correspondant aux périodes de retour données, calculées en chaque observatoire ont permis de tracer les courbes de niveau. Un exemple en est donné sur la figure 4, correspondant à une période de retour de 10 ans.

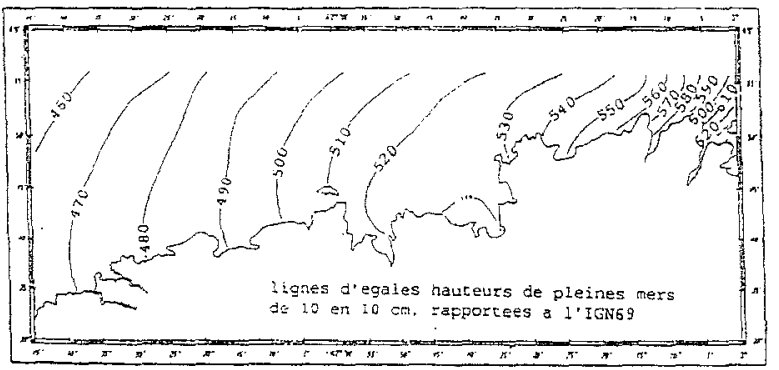

figure 4 
La zone située au sud des Sables-d'Olonne n'a pas été étudiée car les mesures marégraphiques n'ont pas été jugées suffisamment nombreuses, en particulier dans les Pertuis et au sud de la pointe de Grave.

\section{PRECISION}

Le problème de la précision se pose différemment selon qu'il s'agit des ports de reférence ou des ports secondaires. Mais dans les deux cas, seule la loi de répartition des surcotes peut influer sur la précision des résultats puisque les défauts éventuels de la prédiction se répercutent intégralement sur les surcotes. La seule restriction concerne la sensibilité plus ou moins grande du résultat final ou modèle de répartition des surcotes.

Concernant la répartition des surcotes, des incertitudes peuvent résulter du choix de la loi de répartition. Or, il a été établi qu'une modification brutale de la loi de Gumbel pour les valeurs de surcote élevées n'avait qu' un effet faible sur les résultats. Nous admettrons que les résultats obtenus pour les ports principaux ont un degré de précision acceptable, bien qu'il ne soit pas réellement possible de l'evaluer.

Pour les ports secondaires, la loi de répartition des surcotes dépend des paramètres de la droite de régression liant les surcotes au port de référence et au port secondaire. Hors des points d'observation, elle dépend également de l'interpolation spatiale.

Les incertitudes sur les hauteurs pour une période de retour donnée ont été évaluées à partir de la stabilité des résultats de l'interpolation en affectant un poids, fonction du coefficient de détermination, à chaque port secondaire. Ces poids sont utilisés dans le modèle d'interpolation pour le contraindre à coïncider plus ou moins avec les résultats obtenus aux ports secondaires.

La comparaison des courbes obtenues permet de situer les zones d'instabilite où des mesures complémentaires sont requises.

Dans les zones correspondant à la Rade de Brest, la Manche-est et le Pas de Calais sont toujours inférieures à $5 \mathrm{~cm}$.

Certaines zones font paraittre des valeurs relativement élevées. C'est le cas, par exemple, du fond de la Baie de Saint-Brieuc.

\section{CONCLUSION}

Cette étude a montré la possibilité de déterminer les hauteurs de marée atteintes le long des côtes de France avec une période de retour donnée. Les résultats, présentés sous forme de cartes doivent être considerés comme les meilleures estimations possibles actuellement compte tenu des données disponibles. Des améliorations sensibles devraient être obtenues dans certaines zones (baie de Saint-Brieuc, côte des Landes) après l'exploitation de nouvelles mesures à réaliser.

La méthode a été mise au point à partir des données disponibles sur les côtes françaises de la Mer du Nord, La Manche et l'Atlantique où la densité d'observatoires de marée est exceptionnellement élevée, ce qui a permis à partir d'interpolations, de tracer les lignes d'égales hauteurs. Il a été établi cependant que 
dans certaines zones, des observations supplementaires sont souhaitables. L'application de cette méthode à d'autres zones devrait donc être précédée d'une campagne de mesures marégraphiques.

Ces résultats destinés à aider à la délimitation du Domaine Public Maritime seront également très utiles pour d'autres applications, et en particulier pour les aménagements portuaires.

Ce travail a été réalisé pour le compte de la Direction des Ports et de la Navigation maritime dans le cadre de l'étude de la proposition de loi de Monsieur Raymond MARCELLIN, Député, relative au domaine public maritime. 


\section{BRLIOGRAPHIE}

LENNON G.W. A frequency investigation of abnormally high tidal levels at certain west coast ports.

- Proceedings of the Institution of Civil Engineers, 25, August 1963. - p. 451-484.

GRAFF J. An Investigation of the frequency distribution of annual sea level maxima at ports around Great Britain.

- Estuarine, Coastal and Shelf Science, 12, 1981. - p. 389-449.

WALDEN A.T. Approximate confidence intervals for port diagram curves.

PRESCOTT P. - Proceedings of the Institution of Civil Engineers, part 2,69, December 1980. - p. 1015-1023.

MDDLETON J.F. Return Periods of extreme sea levels from short records.

THOMSON K.R. - Journal of Geophysical Research, Vol. 91, NO CIO, 15, October 1986. - p 11707-11716

SUTHONS C.T. Frequency of occurrence of abnormally high sea levels on the east and south coasts of England.

- Proceedings of the Institution of Civil Engineers, 25, August 1963. - p. 433-449.

PUGH D.T. Extreme sea levels form tide an surge probability.

VASSIE J.M. - Proceedings of the 16th Coastal Engineering Conference, Hamburg, 1978. American Society of Civil Engineers, New York, 1979. - p. 911 930.

TAWN J.A. Extreme sea levels. The joint probabilities method revisited and revised. VASSIE J.M. - Proceedings of the Institution of Civil Engineers, part 2, 87, September1989. - p. 429-442.

TAWN J.A. Spatial transfer of extreme sea level data for use in the revised joint probability method.

VASSIE J.M. - Proceedings of the Institution of Civil Engineers, part 2, 89, September 1990 . - p. 433-438.

TAWN J.A. Recent improvement in the joint probability method for estimating extreme sea levels.

VASSTE J.M. - Tidals Hydrodynamics, National Ocean Service, National Oceanic and Atmospheric Administration, US Department of Commerce, 41, 1991. - p. 813827.

SMON B. Calcul de la marée au large pour la réduction des sondages.

- Revue Hydrographique Internationale, Monaco, LXVII(2), juillet 1990.

SMON B. Species concordance method of tide prediction.

- Hydrodynamic Bruce B. Parker (ed.), NOS, NOAA, US Department of Commerce, 1991.

GUMBEL E.J. Statistics of extremes

- Columbia University Press, New York, 1958.

DUCHON J. Interpolation des fonctions de deux variables suivant le principe de la flexion des plaques minces.

- Revue française d'automatique, informatique, recherche operationnelle, série analyse numérique, Vol. $10, \mathrm{n}^{\circ} 12$, décembre 1976. - p. 5-12. 